Research Article

\title{
A Model Developed for Predicting Uniformity of Kyphoplasty Balloon Wall Thickness Based on the Orthogonal Test
}

\author{
Guanghui Dai $\mathbb{i}$, Qingqing Zhang, and Guobao Jin \\ College of Mechanical Engineering, Chaohu University, Chaohu, Anhui 238000, China \\ Correspondence should be addressed to Guanghui Dai; daiguanghui1987@126.com
}

Received 9 February 2020; Revised 8 May 2020; Accepted 16 May 2020; Published 29 May 2020

Academic Editor: Georgios I. Giannopoulos

Copyright ( $) 2020$ Guanghui Dai et al. This is an open access article distributed under the Creative Commons Attribution License, which permits unrestricted use, distribution, and reproduction in any medium, provided the original work is properly cited.

\begin{abstract}
In order to optimize the wall thickness distribution of medical balloon, kyphoplasty balloon was chosen as the research object, the uniformity of wall thickness distribution was taken as the evaluation index, and the influence of stretch blow molding process on the uniformity of kyphoplasty balloon was investigated. In this paper, 16 sets of orthogonal test schemes were studied by selecting four main parameters such as forming temperature, forming pressure, stretching distance, and holding time of stretch blow molding process based on the $L_{16}\left(4^{4}\right)$ Taguchi method orthogonal table. The statistical analysis showed that the forming temperature was an utmost parameter on the uniformity, while an optimal scheme was obtained and an optimal balloon with the uniformity of $95.86 \%$ was formed under the scheme. To further quantify the relationship between the uniformity and the parameters, artificial neural network (ANN) and nonlinear regression (NLR) models were developed to predict the uniformity of the balloon based on orthogonal test results. A feed-forward neural network based on backpropagation (BP) was made up of 4 input neurons, 11 hidden neurons, and one output neuron, an objective function of the NLR model was developed using secondorder polynomial, and the BFGS method was used to solve the function. Adequacy of models was tested using hypothesis tests, and their performances were evaluated using the $R^{2}$ value. Results show that both predictive models can be used for predicting the uniformity of the balloon with a higher reliability. However, the NLR model showed a slightly better performance than the ANN model.
\end{abstract}

\section{Introduction}

Medical balloon is a device used for interventional surgery and has been widely used in many medical fields [1]. The balloon has extremely demanding performance requirement, and great attention has been paid to the balloon forming process investigation to form high-quality products. Sauerteig and Giese [2] described that balloon is manufactured by stretch blow molding process using the extruded tube, which is the basic method of balloon manufacturing; they also researched the influence of process parameters on the manufacture of PTCA balloon and defined the pressure and diameter characteristics. Some improved manufacturing processes based on stretch blow molding are documented on patents [3-5]. Menary and Armstrong [6] researched the forming process of angioplasty balloon through numerical modeling and simulation and developed a finite element simulation of the process to optimize the design and manufacture of the balloon. Azarnoush et al. [7] assessed the diameter of angioplasty balloon at different pressures through coherence tomography monitoring. Lalli [8] researched the relationship between wall thickness and four main stretch blow molding parameters and expounded the relationship between burst pressure, wall thickness, and compliance. Fu et al. [9] analyzed the influence of process parameters on wall thickness through numerical simulation based on the orthogonal design method and established the regression model of wall thickness. Mir et al. [10] simulated the stretch blow molding process using the pressure-volume thermodynamic relationship to predict the thickness of the balloon. Ro and Davé [11] researched the effects of critical design attributes of angioplasty balloons, such as residual stresses on shrinkage, crystallite orientation, balloon compliance, and mechanical properties. 
To summarize, there are some researches on the forming processes for optimizing balloon characteristics such as pressure, wall thickness, and compliance. However, another important performance of the balloon, the uniformity of wall thickness distribution, is rarely studied. This performance not only limits burst pressure but also affects flexibility. The reason is that the thicker the balloon wall, the greater the bursting pressure, and the worse the flexibility, and if the balloon is too thin, the burst pressure will be low.

\section{Kyphoplasty Balloon Production and Measurement}

2.1. Kyphoplasty Balloon. Like other typical medical balloons, a kyphoplasty balloon consists of five descriptive areas, namely one body, two cones, and two necks. The body is a smooth cylindrical part to restore the vertebral height lost during the operation, the necks are extreme ends which are used to connect with the catheter, and the cones are conical parts between the body and the necks. Figure 1 illustrates the structure and size of kyphoplasty balloon researched in this paper.

2.2. Material. Kyphoplasty balloon is a compliant balloon, and its volume is able to expand to several times their original size and usually return back to their original shape and size once the pressure is reduced [12]. In order to meet the performance requirements, thermoplastic polyurethane $(T P U)$ is usually used as the material for manufacturing the balloon. In this paper, a new type of aromatic polyetherbased TPU (RxT90A; Texin ${ }^{\circledR}$, Germany) with a melting point of $199^{\circ} \mathrm{C}$ and a Vicat softening temperature of $106^{\circ} \mathrm{C}$ was selected for forming experiments.

2.3. Forming Process. The forming process of kyphoplasty balloon usually includes three main phases. First, the tube with an inner diameter of $3.5 \mathrm{~mm}$ and an outer diameter of $4.3 \mathrm{~mm}$ is extruded, and it must have a high concentricity and a uniform wall thickness. Second, a parison is achieved when both ends of the tube are stretched at a high temperature and an unstretched segment is left in the middle. Third, the balloon is formed in stretch blow molding process, which is the phase of research in this paper. In this phase, the tube with a parison passes through center mold and two ends of the tube are placed in the plugs, respectively, followed by axial prestretching of both ends at low pressure; then, a balloon is formed when the parison is extended and expanded inside the mold as the pressure raised after mold heating and keep warming for a while; at the end of this process, the mold is cooled down and the pressure is released.

Both of the parison and balloon are formed in a balloon forming system, which includes a double-end stretcher (DES) and a balloon-forming machine (BFM). Figure 2 illustrates a balloon forming system (CMBPL-01; Changmei Medtech, China).

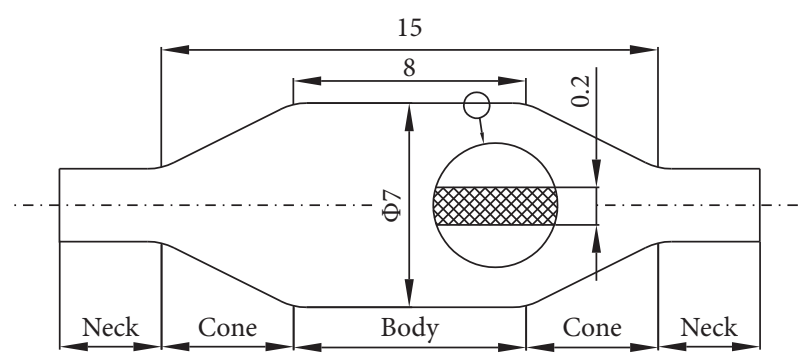

FIGURE 1: Structure and size of kyphoplasty balloon.

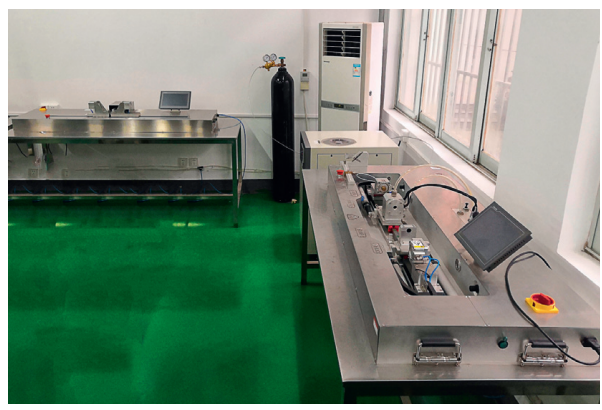

Figure 2: Balloon forming system: DES (left) and BFM (right).

2.4. Measurement and Characterization. Five continuously produced balloons were taken as the samples, and each sample was marked with 7 positions along axial direction, as shown in Figure 3. Three sets of double-wall thickness data were measured along a circle at each position, and the result of the sum of one-sixth of the three data at one position is taken as the result. The mean of the results at the same position of the five samples was calculated as the experimental result on each position, regarded as $\delta_{i}(i=0,1, \ldots, 7)$. All measurements were measured using the micrometer.

The uniformity of kyphoplasty balloon wall thickness distribution is developed as the following objective function [13]:

$$
G=100 \%-\frac{S_{t d}}{\bar{\delta}} \times 100 \%
$$

where $G$ is the uniformity of wall thickness, $S_{t d}$ is the standard deviation of wall thickness, $\bar{\delta}$ is the mean of wall thickness, and $S_{t d}$ and $\bar{\delta}$ can be calculated according to the following two formulas:

$$
\begin{aligned}
S_{t d} & =\left[\sum_{i=1}^{n} \frac{\left(\delta_{i}-\bar{\delta}\right)^{2}}{n}\right]^{1 / 2}, \\
\bar{\delta} & =\frac{1}{n}\left(\delta_{1}+\delta_{2}+\cdots+\delta_{n}\right) .
\end{aligned}
$$

\section{Orthogonal Test and Results}

3.1. Orthogonal Test Factors. Orthogonal test is an efficient, fast, and economical method for seeking the best level combination with multiple factors [14]. Some important test factors and representative level combinations are selected in 


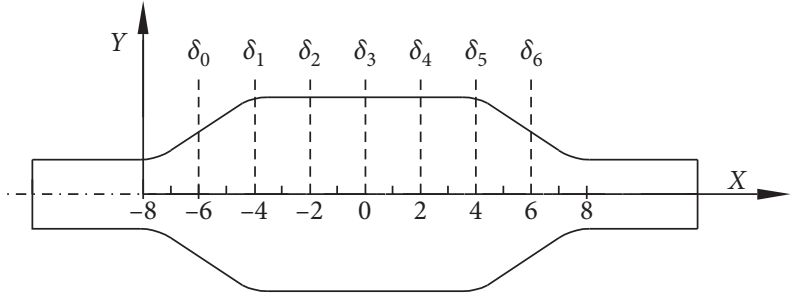

Figure 3: Marked positions.

order to find the optimal and effective level combination. It can be concluded from previous studies [8-10] that forming temperature $(Q)$, forming pressure $(P)$, stretching distance $(D)$, and holding time $(T)$ are four important factors in stretch blow molding process. In this paper, the above four factors are selected as test factors, and each factor is set with four different levels according to the appropriate range of preliminary tests, as shown in Table 1. In preliminary tests, some other stretch blow molding parameters were determined, such as the length of parison, $10 \mathrm{~mm}$, the cooling water temperature, $23^{\circ} \mathrm{C}$, the cooling time, $90 \mathrm{~s}$, the prestretching force, $80 \mathrm{~g}$, and the initial pressure, $2 \mathrm{~atm}$.

3.2. Orthogonal Test Schemes. The Taguchi orthographic table was selected to design the test schemes based on Table 1 $[15,16] .16$ sets of orthogonal test schemes were established according to the $L_{16}\left(4^{4}\right)$ orthogonal table, as shown in Table 2.

3.3. Orthogonal Test Results. Balloons were formed according to the schemes, and the uniformity of the balloon wall thickness under each scheme was measured and calculated according to formulas (1)-(3). The full orthogonal test results are shown in Table 3.

3.4. Range Analysis. There are two important parameters, $K_{i}$ and $\Re$, in range analysis, and they are described as shown in the following equation [17]:

$$
\begin{aligned}
& K_{i}=\frac{1}{n} \sum_{j=1}^{n} E_{j}, \\
& \mathfrak{R}=K_{i \max }-K_{i \min },
\end{aligned}
$$

where $E_{j}$ is the value of a certain factor, $K_{i}$ is the average of each factor, and $\mathfrak{R}$ is the range and used to estimate design variable sensitivities [18]. In general, the greater the $K_{i}$ value, the higher the indicator values under this level, and if the value of $\Re$ is larger, the factor value would be more influential [19]. The range analysis of the orthogonal test results was calculated according to equation (4), as shown in Table 4.

It can be clearly found from Table 4 that the primary and secondary order of influence of each parameter on the uniformity of wall thickness is BADC. The optimal combination of the uniformity as the evaluation criterion is $A_{3} B_{3} C_{3} D_{2}$, namely, $P=6 \mathrm{~atm}, Q=160^{\circ} \mathrm{C}, T=40 \mathrm{~S}$, and
TABLE 1: Level table of orthogonal test factors.

\begin{tabular}{lcccc}
\hline Levels & \multicolumn{4}{c}{ Factors } \\
& $\begin{array}{c}A \\
P(\mathrm{~atm})\end{array}$ & $\begin{array}{c}B \\
\left({ }^{\circ} \mathrm{C}\right)\end{array}$ & $\begin{array}{c}C \\
T(\mathrm{~s})\end{array}$ & $\begin{array}{c}D \\
D(\mathrm{~mm})\end{array}$ \\
\hline 1 & 4 & 140 & 20 & 8 \\
2 & 5 & 150 & 30 & 10 \\
3 & 6 & 160 & 40 & 12 \\
4 & 7 & 170 & 50 & 14 \\
\hline
\end{tabular}

\begin{tabular}{|c|c|c|c|c|c|c|c|}
\hline \multirow{2}{*}{$\begin{array}{l}\text { Test serial } \\
\text { numbers }\end{array}$} & \multicolumn{3}{|c|}{ Factors } & \multicolumn{4}{|c|}{ Corresponding parameters } \\
\hline & A & $B$ & $C I$ & $\begin{array}{c}P \\
(\mathrm{~atm})\end{array}$ & $\begin{array}{c}Q \\
\left({ }^{\circ} \mathrm{C}\right)\end{array}$ & $\begin{array}{c}T \\
(\mathrm{~s})\end{array}$ & $\underset{(\mathrm{mm})}{D}$ \\
\hline 1 & $A_{1}$ & $B_{1}$ & $C_{1} D$ & 4 & 140 & 20 & 8 \\
\hline 2 & $A_{1}$ & $B_{2}$ & $C_{2} \mathrm{D}$ & 4 & 150 & 30 & 10 \\
\hline 3 & $A_{1}$ & $B_{3}$ & $C_{3} \mathrm{D}$ & 4 & 160 & 40 & 12 \\
\hline 4 & $A_{1}$ & $B_{4}$ & $C_{4} D$ & 4 & 170 & 50 & 14 \\
\hline 5 & $A_{2}$ & $B_{1}$ & $C_{2} \mathrm{D}$ & 5 & 140 & 30 & 12 \\
\hline 6 & $A_{2}$ & $B_{2}$ & $C_{1} \quad D$ & 5 & 150 & 20 & 14 \\
\hline 7 & $A_{2}$ & $B_{3}$ & $C_{4} \mathrm{D}$ & 5 & 160 & 50 & 8 \\
\hline 8 & $A_{2}$ & $B_{4}$ & $C_{3} \mathrm{D}$ & 5 & 170 & 40 & 10 \\
\hline 9 & $A_{3}$ & $B_{1}$ & $C_{3} \mathrm{D}$ & 6 & 140 & 40 & 14 \\
\hline 10 & $A_{3}$ & $B_{2}$ & $C_{4} \mathrm{D}$ & 6 & 150 & 50 & 12 \\
\hline 11 & $A_{3}$ & $B_{3}$ & $C_{1} D$ & 6 & 160 & 20 & 10 \\
\hline 12 & $A_{3}$ & $B_{4}$ & $C_{2} \mathrm{D}$ & 6 & 170 & 30 & 8 \\
\hline 13 & $A_{4}$ & $B_{1}$ & $C_{4} \mathrm{D}$ & 7 & 140 & 50 & 10 \\
\hline 14 & $A_{4}$ & $B_{2}$ & $C_{3} D$ & 7 & 150 & 40 & 8 \\
\hline 15 & & $B_{3}$ & $C_{2} \quad D$ & 7 & 160 & 30 & 14 \\
\hline 16 & $A_{4}$ & $B_{4}$ & $C_{1} \quad D$ & 7 & 170 & 20 & 12 \\
\hline
\end{tabular}

TABLE 2: Orthogonal test schemes.

TABle 3: Orthogonal test results.

\begin{tabular}{lc}
\hline Test serial numbers & $G$ \\
\hline 1 & $80.95 \%$ \\
2 & $87.22 \%$ \\
3 & $89.47 \%$ \\
4 & $83.51 \%$ \\
5 & $83.76 \%$ \\
6 & $88.18 \%$ \\
7 & $93.74 \%$ \\
8 & $93.67 \%$ \\
9 & $85.69 \%$ \\
10 & $92.58 \%$ \\
11 & $95.26 \%$ \\
12 & $93.27 \%$ \\
13 & $85.51 \%$ \\
14 & $90.27 \%$ \\
15 & $92.13 \%$ \\
16 & $90.23 \%$ \\
\hline
\end{tabular}

TABLE 4: Influence analysis of parameters on the uniformity.

\begin{tabular}{lcccc}
\hline Parameters & $P(\mathrm{~atm})$ & $Q\left({ }^{\circ} \mathrm{C}\right)$ & $T(\mathrm{~s})$ & $D(\mathrm{~mm})$ \\
\hline$K_{1}$ & $85.29 \%$ & $83.99 \%$ & $88.66 \%$ & $89.83 \%$ \\
$K_{2}$ & $89.84 \%$ & $88.56 \%$ & $89.10 \%$ & $90.22 \%$ \\
$K_{3}$ & $91.70 \%$ & $92.65 \%$ & $89.78 \%$ & $89.11 \%$ \\
$K_{4}$ & $89.54 \%$ & $90.17 \%$ & $88.84 \%$ & $88.20 \%$ \\
$R$ & $6.41 \%$ & $8.67 \%$ & $1.12 \%$ & $3.03 \%$ \\
Sorting & 2 & 1 & 4 & 3 \\
\hline
\end{tabular}


$D=10 \mathrm{~mm}$. The poor combination of the uniformity as the evaluation criterion is $A_{1} B_{1} C_{1} D_{4}$, namely, $P=4 \mathrm{~atm}$, $Q=140^{\circ} \mathrm{C}, T=20 \mathrm{~S}$, and $D=14 \mathrm{~mm}$.

\section{Development of Predictive Models}

In order to quickly obtain appropriate process conditions, the method of directly predicting test results with the help of numerical simulation is a simple and economical method for enterprises [20]. It is necessary, for this purpose, to establish a mathematical model for quantifying the relationship between the uniformity and main parameters.

4.1. Artificial Neural Networks (ANN). ANN became more and more popular in the recent past for the development of process parameter predictive models [21-23]. This method is an efficient modeling technique that can capture and generate both linear and nonlinear complex relationships between the independent and dependent variables [24]. In this paper, a feed-forward backpropagation (BP) algorithm was used to model the wall thickness uniformity of the balloon. The network has three layers, namely, input layer, output layer, and hidden layer. There are four neurons in the input layer, namely, forming temperature, forming pressure, stretching distance, and holding time, and there is one neuron in the output layer, namely, the wall thickness uniformity. The hidden layer performs nonlinear mapping between input and output layers through a suitable basis function, and the number of neurons in this layer can be calculated by using the following formula [25]:

$$
s=\sqrt{m+k}+a,
$$

where $s$ is the number of hidden neurons, $m$ is the number of input neurons, $k$ is the number of output neurons, and $a$ is a constant between 0 and 10 . The neurons of the hidden layer are determined to be 11 . The architecture of this 4-11-1 network is shown in Figure 4.

The training, testing, and validation of the ANN analysis were conducted using MATLAB, 2017a (The MathWorks, Inc.). Based on the recommendations of Kurra et al. [26], $95 \%$ of the data were used for training, $5 \%$ of the data were used for testing, and $5 \%$ of the data were used for validation.
The network was trained using the Levenberg-Marquardt function, which was found to be an efficient training function $[26,27]$.

\subsection{Regression Model and Calculation}

4.2.1. Regression Model. A nonlinear objective function $G(x)$ was established considering the nonlinear relationship between the uniformity and main parameters, and the variables of the model were assumed such as forming temperature, forming pressure, stretching distance, holding time, and the wall thickness uniformity. According to the studies [28, 29], second-order polynomial function can better represent the relationship between process conditions and assessment indicators than first-order function, and the amount of fitting calculation required for the second-order polynomial function is less than the third-order and higherorder polynomial functions. The objective function can be defined as follows:

$$
G(x)=\beta_{0}+\sum_{i=1}^{k} \beta_{i} X_{i}+\sum_{i=1}^{k} \beta_{i i} X_{i}^{2}+\sum \sum_{i<j} \beta_{i j} X_{i} X_{j}+\varepsilon_{i},
$$

where $k$ is number of factors, $\beta_{0}$ is the free term, $\beta_{i}$ is the linear effect, $\beta_{i i}$ is the squared effect, and $\beta_{i j}$ is the interaction effect.

4.2.2. Calculation Based on BFGS. The quasi-Newton method is an effective method for solving nonlinear function [30] and is proved very successful based on the numerical experiment [31]. In this paper, the BFGS method, a branch of quasi-Newton methods, was used to solve function (6). The solution and convergence steps based on the BFGS algorithm were illustrated by Nocedal and Wright [32]. The nonlinear regression (NLR) model, described as follows, was obtained after calculation according to the parameter combinations in Table 3 and the corresponding test results in Table 4 with the help of the mathematical analysis software 1stOpt:

$$
\begin{aligned}
G(x)= & f(Q, P, D, T)=0.88384389-76.517016 Q+2.1043957 P+27.562052 D-5.9743062 T \\
& +0.00048819 Q \cdot P+2.778543 Q \cdot D-6.93864 e-5 Q \cdot T-0.0733895 P \cdot D-0.00102068 P \cdot T \\
& +0.217053 D \cdot T-0.000201625 Q^{2}-0.0167875 P^{2}-1.0074298 D^{2}-3.45 e-5 T^{2}
\end{aligned}
$$

4.3. Results and Discussion. The predictive models of the wall thickness uniformity were developed as a function of forming temperature, forming pressure, stretching distance, and holding time. There are two different techniques-ANN and NLR - that can be used to develop these models for the estimation of the wall thickness distribution of the balloon. Results of ANN and NLR are compared with experiments in Table 3 for 16 check sets, and their relative errors are calculated between predicted and experimental values. The comparison results and errors are depicted in Table 5.

The results predicted by ANN and NLR are compared with experimental results in Figure 5. From this figure, it can be seen that both models' prediction present good agreement with the experimental data.

Performances of these techniques were evaluated through calculating errors. The statistic of the errors with 


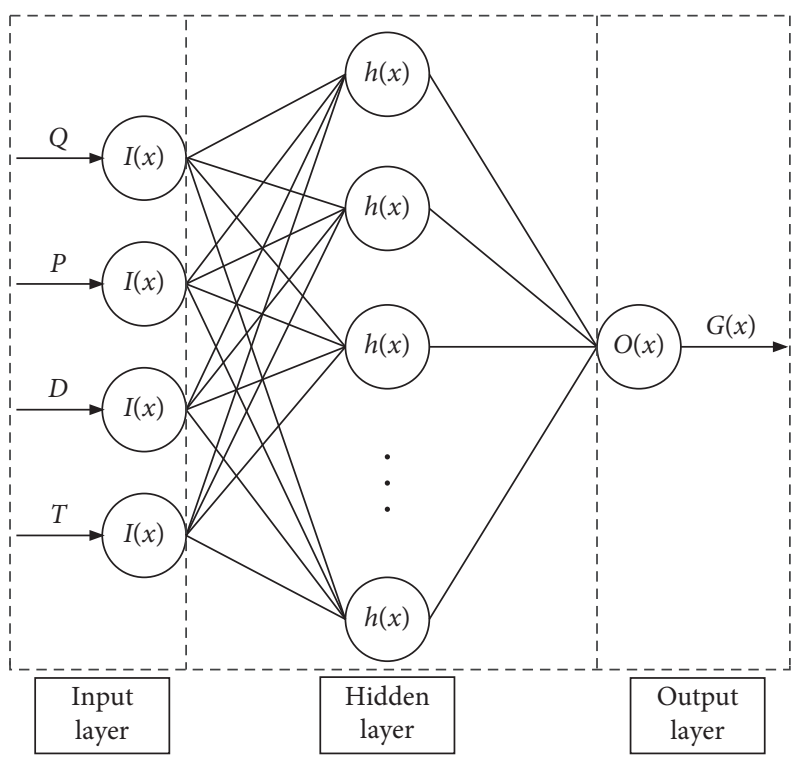

FIGURE 4: Backpropagation network.

TABLE 5: Comparison of ANN and NLR model results with experimental measurements and their relative error.

\begin{tabular}{|c|c|c|c|c|c|}
\hline \multirow{2}{*}{ Test serial numbers } & \multicolumn{3}{|c|}{$G$} & \multicolumn{2}{|c|}{ Error } \\
\hline & Experimental measurements & ANN model & NLR model & ANN model & NLR model \\
\hline 1 & $80.95 \%$ & $80.93 \%$ & $80.16 \%$ & $0.02 \%$ & $0.79 \%$ \\
\hline 2 & $87.22 \%$ & $85.72 \%$ & $87.66 \%$ & $1.50 \%$ & $0.44 \%$ \\
\hline 3 & $89.47 \%$ & $89.39 \%$ & $89.04 \%$ & $0.08 \%$ & $0.43 \%$ \\
\hline 4 & $83.51 \%$ & $84.26 \%$ & $84.31 \%$ & $0.75 \%$ & $0.80 \%$ \\
\hline 5 & $83.76 \%$ & $84.27 \%$ & $83.87 \%$ & $0.51 \%$ & $0.11 \%$ \\
\hline 6 & $88.18 \%$ & $87.40 \%$ & $90.01 \%$ & $0.78 \%$ & $1.83 \%$ \\
\hline 7 & $93.74 \%$ & $90.98 \%$ & $93.00 \%$ & $2.76 \%$ & $0.74 \%$ \\
\hline 8 & $93.67 \%$ & $93.42 \%$ & $92.40 \%$ & $0.25 \%$ & $1.27 \%$ \\
\hline 9 & $85.69 \%$ & $84.05 \%$ & $85.50 \%$ & $1.64 \%$ & $0.19 \%$ \\
\hline 10 & $92.58 \%$ & $91.74 \%$ & $93.32 \%$ & $0.84 \%$ & $0.74 \%$ \\
\hline 11 & $95.26 \%$ & $92.89 \%$ & $93.44 \%$ & $2.37 \%$ & $1.82 \%$ \\
\hline 12 & $93.27 \%$ & $93.52 \%$ & $94.49 \%$ & $0.25 \%$ & $1.22 \%$ \\
\hline 13 & $85.51 \%$ & $86.49 \%$ & $85.28 \%$ & $0.98 \%$ & $0.23 \%$ \\
\hline 14 & $90.27 \%$ & $90.31 \%$ & $90.34 \%$ & $0.04 \%$ & $0.07 \%$ \\
\hline 15 & $92.13 \%$ & $90.74 \%$ & $92.06 \%$ & $1.39 \%$ & $0.07 \%$ \\
\hline 16 & $90.23 \%$ & $91.89 \%$ & $90.45 \%$ & $1.66 \%$ & $0.22 \%$ \\
\hline
\end{tabular}

different modeling techniques is summarized in Table 6. It can be known from this table that the mean test errors for ANN and NLR are about $0.99 \%$ and $0.69 \%$, respectively, and the maximum test errors for them are about $2.76 \%$ and $1.83 \%$, respectively. Both methods are suitable for estimating wall thickness uniformity in an acceptable error range. However, both of the mean error and maximum error in NLR was found to be less compared with ANN.

Three hypothesis tests, namely, mean paired $t$-test, F-test, and Levene's test, were conducted to test the goodness of fit. Results of the hypothesis tests of two modeling techniques (ANN and NLR) were compared with the significance level 0.05 in Figure 6. From this figure, it can be known that the calculated $P$ value with three hypothesis tests are greater than 0.05 . This indicates that there is no significant difference between experimental and predicted values using ANN and NLR.
The validity of the ANN and NLR models have been tested with correlation coefficient $R^{2}$ value, which is calculated using [27]:

$$
R^{2}=1-\frac{\sum\left(y_{i}-f_{i}\right)^{2}}{\sum\left(y_{i}-\bar{y}\right)^{2}},
$$

where $y_{i}, f$, and $\bar{y}$ are experimental, predicted, and mean values of wall thickness uniformity, respectively. The $R^{2}$ values of wall thickness uniformity with ANN and NLR were 0.945 and 0.954 , respectively. For the adequacy of model, $R^{2}$ value should be between 0.8 and 1 . The high $R^{2}(>0.94)$ value indicated that the developed model can be used to predict. The $R^{2}$ values of the two methods were higher than 0.94 , which indicated that the presented model fits the data very well. However, the $R^{2}$ values for the NLR model were smaller than that for ANN. 


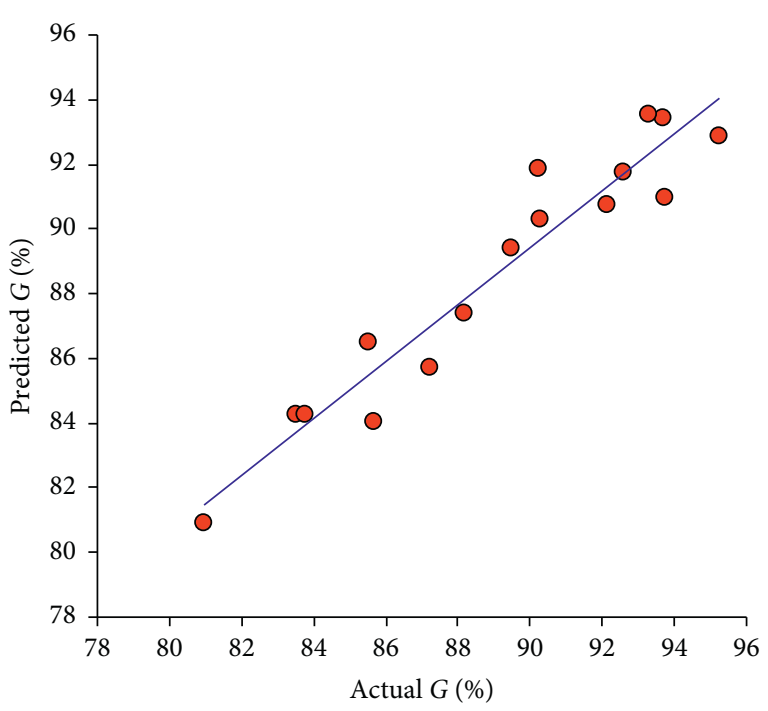

(a)

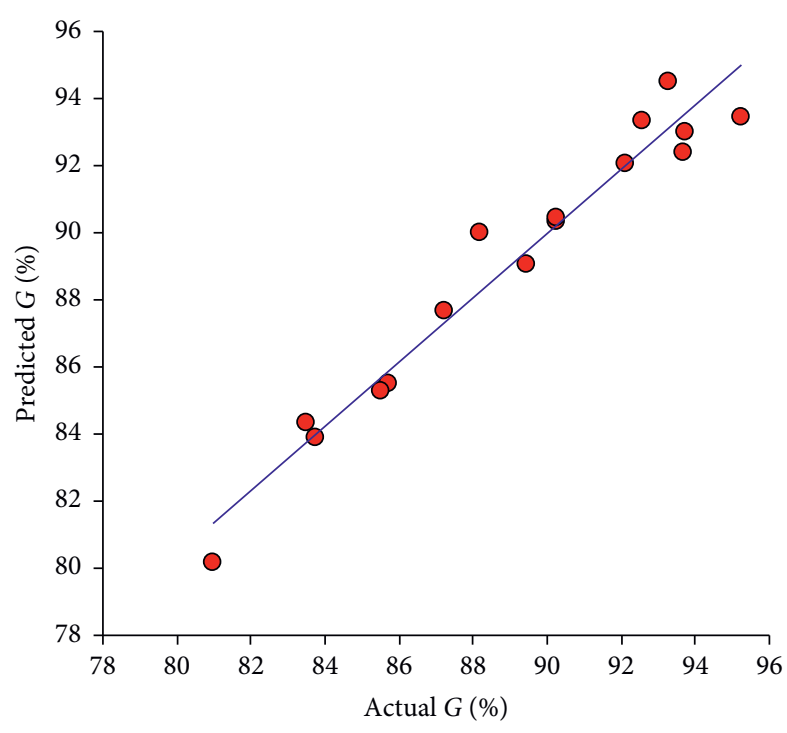

(b)

FIgure 5: Predicted and actual values of $G$ using (a) ANN and (b) NLR.

TABLE 6: Error statistics with ANN and NLR.

\begin{tabular}{lccccc}
\hline Model & Count & Mean & Std Dev & Minimum & Maximum \\
\hline ANN & 16 & $0.99 \%$ & 0.0083 & $0.02 \%$ & $2.76 \%$ \\
NLR & 16 & $0.69 \%$ & 0.0059 & $0.07 \%$ & $1.83 \%$ \\
\hline
\end{tabular}

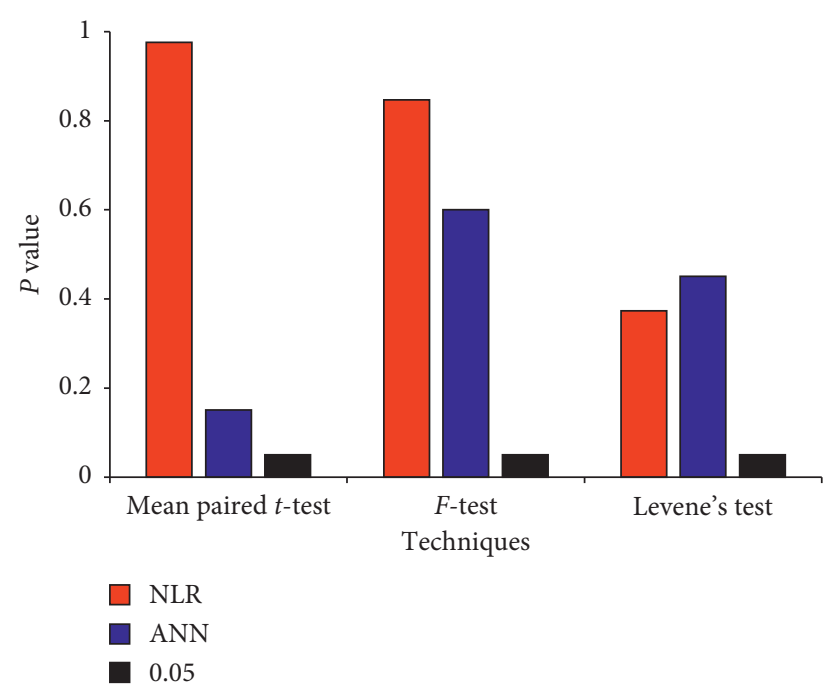

Figure 6: Descriptive statistics of hypothesis tests.

4.4. Model Validation. To verify the practicability of the ANN and NLR models, two parameter combinations, the optimal combination $A_{3} B_{3} C_{3} D_{2}$ and the poor combination $A_{1} B_{1} C_{1} D_{4}$ that obtained from the range analysis, were simulated and experimented. Results of the ANN and regression analysis were compared with experiments for 2 check sets, and their relative errors were calculated between predicted and experimental values. The comparison results and errors are depicted in Table 7 . From this table, it can be known that the predicted errors for ANN and NLR are about $0.06 \%$ and $0.34 \%$, respectively, in the poor combination $A_{1} B_{1} C_{1} D_{4}$ and the errors are about $1.73 \%$ and $0.28 \%$, respectively, in the optimal combination $A_{3} B_{3} C_{3} D_{2}$. It indicates that both methods are suitable for estimating wall 
TABLE 7: Comparison results and their relative errors of two combinations.

\begin{tabular}{lccccc}
\hline Combination & $G$ & & \multicolumn{2}{c}{ Error } & \\
& Experimental measurements & Regression model & ANN model & ANN model & Regression model \\
\hline$A_{1} B_{1} C_{1} D_{4}$ & $79.37 \%$ & $79.03 \%$ & $79.31 \%$ & $0.06 \%$ & $0.34 \%$ \\
$A_{3} B_{3} C_{3} D_{2}$ & $95.86 \%$ & $95.58 \%$ & $94.13 \%$ & $1.73 \%$ & $0.28 \%$ \\
\hline
\end{tabular}

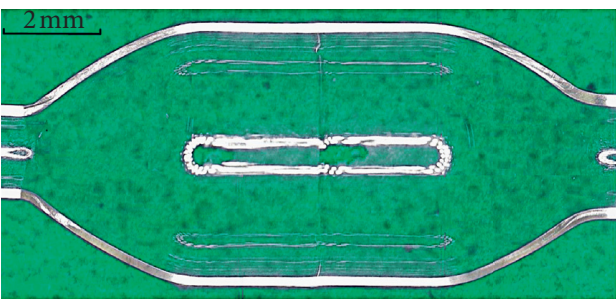

(a)

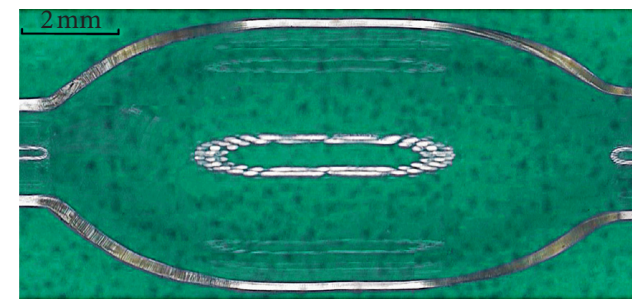

(b)

Figure 7: Cross-sectional picture of balloon: (a) optimal balloon and (b) poor balloon.

thickness uniformity in an acceptable error range, and the two models can be used to express the relationship between wall thickness uniformity and main parameters for balloon forming. Nevertheless, the maximum error occurred in the ANN model, and both of the mean error and maximum error in NLR were found to be less compared with ANN. This comparison results were consistent with previous 16 check sets. The model generation and training procedure of the ANN model took more time than that of the regression model, and the same conclusion is described in the study by Caydas and Hascalik [28]. These reveals that the NLR techniques have better performance than ANN. Figure 7 illustrates the cross-sectional picture of balloons that are randomly selected from the two groups.

\section{Conclusions}

To optimize the wall thickness distribution of medical balloon, the influence of stretch blow molding process on the wall thickness uniformity of kyphoplasty balloon was investigated. The 16 sets of orthogonal test schemes were researched based on the $L_{16}\left(4^{4}\right)$ Taguchi method orthogonal table by selecting the four main parameters-forming pressure $(P)$, forming temperature, stretching distance, and holding time of the process. An optimal scheme with the forming pressure of $6 \mathrm{~atm}$, the forming temperature of $160^{\circ} \mathrm{C}$, the stretching distance of $10 \mathrm{~mm}$, and the heating time of $40 \mathrm{~s}$ was obtained; and an optimal balloon with the uniformity $95.86 \%$ was formed under the scheme. Based on the range analysis, the most dominant parameter of the wall thickness uniformity was forming temperature, the second ranking factor was forming pressure, and stretching distance and holding time were less effective on wall thickness uniformity. In addition, to ascertain the relationship between forming parameters and wall thickness uniformity of medical balloon formed by stretch blow molding process, an ANN analysis and an NLR analysis were carried out based on the orthogonal test results. Comparisons were made of the above approaches after testing their performances on all 16 test cases; both ANN and NLR approaches were sufficient for estimating wall thickness uniformity of medical balloon with in an acceptable error range. Adequacy of models was tested using hypothesis tests, and performance of models was evaluated by using $R^{2}$ value. Results showed that both predictive models can be used for predicting wall thickness uniformity of medical balloon in stretch blow molding process with a higher reliability, and the predicted results were found to be close with the actual performance results. However, the NLR model showed a slightly better performance than the ANN model.

\section{Data Availability}

The data used to support the findings of this study are available from the corresponding author upon request.

\section{Conflicts of Interest}

The authors declare that they have no conflicts of interest.

\section{Acknowledgments}

This work was supported by the Provincial Natural Science Foundation of Anhui Universities (Grant no. KJ2015A225).

\section{References}

[1] L. X. Xu, C. L. Lu, and X. Y. Li, "The research progress of the medical balloon materials and forming techniques," Journal of Dalian University, vol. 31, no. 6, pp. 81-83, 2010.

[2] K. Sauerteig and M. Giese, "The effect of extrusion and blow molding parameters on angioplasty balloon production," Medical Plastics and Biomaterials, no. 5, pp. 46-53, 1998.

[3] M. Y. Zhang, K. Williams, and J. Skinner, "Catheter balloon forming process," Patent EP20010902039, 2002.

[4] J. Simpson, S. Nelson, and J. Durcan, "Catheter balloon and method for forming same," Patent US20100881733, 2014.

[5] S. M. Jeffry, "Method of making a fiber-reinforced medical balloon," Patent US7641844B2, 2008. 
[6] G. H. Menary and C. G. Armstrong, "Experimental study and numerical modelling of injection stretch blow moulding of angioplasty balloons," Plastics, Rubber and Composites, vol. 35, no. 8, pp. 348-354, 2006.

[7] H. Azarnoush, S. Vergnole, R. Sébastien, B. Boulet, and G. Lamouche, "Optical coherence tomography monitoring of angioplasty balloon inflation in a deployment tester," Review of Scientific Instruments, vol. 81, no. 8, pp. 752-760, 2010.

[8] D. Lalli, "Cycle-to-cycle control of the angioplasty balloon fabrication process," Master's thesis, McGill University, Montreal, Canada, 2010.

[9] X. Fu, H. He, and W. Wang, "Numerical simulation of forming process conditions and wall thickness for balloon," in Proceedings of the International Conference on Intelligent Robotics and Applications, Part III, LNAI 10464, pp. 808-818, Wuhan, China, August 2017.

[10] H. Mir, Z. Benrabah, and F. Thibault, "The use of elasticplastic material model coupled with pressure-volume thermodynamic relationship to simulate the stretch blow molding of polyethylene terephthalate," AIP Conference Proceedings, vol. 908, pp. 331-336, 2007.

[11] A. J. Ro and V. Davé, "Properties of nylon 12 balloons after thermal and liquid carbon dioxide treatments," Materials Science and Engineering: C, vol. 33, no. 2, pp. 909-915, 2013.

[12] C. Rogers, D. Y. Tseng, J. C. Squire et al., "Balloon-artery interactions during stent placement: a finite element analysis approach to pressure, compliance, and stent design as contributors to vascular injury," Circulation Research, vol. 84, no. 4, pp. 378-383, 1999.

[13] G. Jin, M. J. Wang, and D. Y. Zhao, "Experimental investigation of extrusion process of double-lumen micro tube," Journal of Mechanical Engineering, vol. 48, no. 24, pp. 19-27, 2012.

[14] Y. M. Wang, Z. C. Yan, and X. J. Shan, "Optimization of process parameters for vertical-faced polypropylene bottle injection molding," Advances in Materials Science and Engineering, vol. 2018, Article ID 2635084, 9 pages, 2018.

[15] H. U. Yong, H. Bolin, and Y. Hong, "Numerical simulation of semi-solid die casting process of magnesium matrix composite," Chinese Journal of Nonferrous Metals, vol. 20, no. 7, pp. 1260-1266, 2010.

[16] A. Scott and Pardo, Taguchi Methods and Robust Design, Springer International Publishing, Cham, Switzerland, 2016.

[17] Y. Q. Wang and X. W. Huo, "Multi-objective optimization design and performance prediction of centrifugal pump based on orthogonal test," Advances in Materials Science and Engineering, vol. 2018, Article ID 6218178, 10 pages, 2018.

[18] H.-Y. Mi, X. Jing, J. Peng, L.-S. Turng, and X.-F. Peng, "Influence and prediction of processing parameters on the properties of microcellular injection molded thermoplastic polyurethane based on an orthogonal array test," Journal of Cellular Plastics, vol. 49, no. 5, pp. 439-458, 2013.

[19] G. Taguchi, "Performance analysis design," International Journal of Production Research, vol. 16, no. 6, pp. 521-530, 1978.

[20] S. K. Madhavi, N. Hanuman, and R. U. Rao, "Regression model developed using RSM for predicting withstanding pressure of HDPE pipe during extrusion process," Recent Advances in MaterialSciences, vol. 8, pp. 177-185, 2019.

[21] N. H. Alharthi, S. Bingol, A. T. Abbas et al., "Prediction of cutting conditions in turning AZ61 and parameters optimization using regression analysis and artificial neural network," Advances in Materials Science and Engineering, vol. 2018, Article ID 1825291, 10 pages, 2018.
[22] Y. Li, C. Liang, X. Lin et al., "Spring back study on profile flexible 3D stretch-bending process using the neural network," Advances in Materials Science and Engineering, vol. 2019, Article ID 6465196, 9 pages, 2019.

[23] S. Karthiyaini, K. Senthamaraikannan, J. Priyadarshini et al., "Prediction of mechanical strength of fiber admixed concrete using multiple regression analysis and artificial neural network," Advances in Materials Science and Engineering, vol. 2019, Article ID 4654070, 7 pages, 2019.

[24] N. Agami, A. Atiya, M. Saleh, and H. El-Shishiny, "A neural network based dynamic forecasting model for Trend Impact Analysis," Technological Forecasting and Social Change, vol. 76, no. 7, pp. 952-962, 2009.

[25] A. Mirigul, "Reducing shrinkage in injection moldings via the Taguchi, anova and neural network methods," Materials and Design, vol. 31, no. 1, pp. 599-604, 2010.

[26] S. Kurra, N. Hifzur Rahman, S. P. Regalla, and A. K. Gupta, "Modeling and optimization of surface roughness in single point incremental forming process," Journal of Materials Research and Technology, vol. 4, no. 3, pp. 304-313, 2015.

[27] P. S. Ghosal and A. K. Gupta, "Enhanced efficiency of ANN using non-linear regression for modeling adsorptive removal of fluoride by calcined Ca-Al-(NO3)-LDH," Journal of Molecular Liquids, vol. 222, pp. 564-570, 2016.

[28] U. Caydas and A. Hascalik, "A study on surface roughness in abrasive waterjet machining process using artificial neural networks and regression analysis method," Journal of Materials Processing Technology, vol. 202, no. 1-3, pp. 574-582, 2008.

[29] C. Lv, The optimization of process conditionals and perform structural parameters for pet bottles, $\mathrm{PhD}$ thesis, Zhejiang University, Montreal, Canada, 2014.

[30] D. Li and M. Fukushima, "A globally and superlinearly convergent gauss--Newton-based BFGS method for symmetric nonlinear equations," SIAM Journal on Numerical Analysis, vol. 37, no. 1, pp. 152-172, 1999.

[31] Z. Wei, G. Li, and L. Qi, "New quasi-Newton methods for unconstrained optimization problems," Applied Mathematics and Computation, vol. 175, no. 2, pp. 1156-1188, 2006.

[32] J. Nocedal and S. J. Wright, Numerical Optimization, Springer, New York, NY, USA, 1999. 